\title{
DETERMINACION DE LA PREVALENCIA DE CISTICERCOSIS PORCINA EN CUATRO VEREDAS DEL MUNICIPIO DE COYAIMA
}

\author{
Jaime A. Serrano', Fredy H. Prada', Ruben S. Nlicholls', \\ Sofía Duque ${ }^{3}$, Jaime Prada ${ }^{4}$, Myriam C. López ${ }^{5}$
}

\begin{abstract}
Durante el período comprendido entre junio y diciembre de 1992, se recolectaron 117 muestras de suero de porcinos en pie provenientes de las veredas: Totarco Dinde, Totarco Piedras, Doyare La Esmeralda y Lomas de Guaguarco, del municipio de Coyaima, departamento del Tolima, con el objeto de determinar la prevalencia de cisticercosis porcina, detectando anticuerpos contra larva de Taenia solium, mediante ELISA.
\end{abstract}

EI ELISA para el serodiagnóstico de cisticercosis porcina fue estandarizado, determinándose que la concentración óptima de antígeno (extracto de larva de $T$. solium) fue de $5 u \mathrm{~g} / \mathrm{ml}$ y las diluciones óptimas de suero y de conjugado (anti- IgG porcina unida a fosfatasa alcalina) fueron 1:4.00 y 1:3.000 respectivamente.

Los parámetros de la prueba, determinados con base en muestras de porcinos con diagnóstico parasitológico comprobado y de cerdos sanos, fueron: sensibilidad $100 \%$, especificidad: $97,14 \%$, valor predictivo positivo: $90 \%$ y valor predictivo negativo $100 \%$.

La prevalencia de cisticercosis porcina en el municipio de Coyaima fue del $25.64 \%$ (30 muestras positivas de 117 analizadas) y del $37,5 \%, 33,33 \%, 17,39 \%$ y $15,63 \%$ en Doyare La Esmeralda, Totarco Piedras, Totarco Dinde y Lomas de Guaguarco respectivamente.

EI ELISA del estudio es útil tanto para el serodiagnóstico de cisticercosis porcina como para estudios epidemiológicos de prevalencia de ésta, orientados a establecer un control de la parasitosis en los cerdos. La alta prevalencia de cisticercosis porcina encontrada en Coyaima hace necesaria la formulación de medidas de control.

\section{INTRODUCCION}

La teniasis es causada por formas adultas de Taenia en conejos, caninos, felinos, ovinos, bovinos y equinos. La $T$. solium es exclusiva del humano (huésped definitivo) y se establece en su intestino siendo por ello en la naturaleza el responsable de la dispersión de los huevos del helminto.

La cisticercosis es una parasitosis causada por la larva de $T$. solium en los tejidos de sus huéspedes intermediarios, el porcino y el humano (1).

1 Facultad de Medicina Veterinaria y Zootecnia, Universidad del Tolima, Ibagué, Colombia.

2 Médico cirujano, MSc Parasitología Médica. Jefe, Grupo de Parasitología, Instituto Nacional de Salud. Profesor adscrito, Departamento de Microbiología y Parasitología. Facultad de Medicina, Universidad Nacional de Colombia, Santafé de Bogotá, D.C., Colombia.

3 Bióloga, MSc Parasitologia Médica, Grupo de Parasitología, Instituto Nacional de Salud. Santafé de Bogotá, D.C., Colombia.

4 Médico Veterinario y Zootecnista, MSc., Universidad del Tolima, Ibagué, Colombia.

5 Bacterióloga, Grupo de Parasitologia, Instituto Nacional de Salud, Santafé de Bogotá. D.C.. Colombia. 
Las cisticercosis humana y porcina son enfermedades que pueden encontrarse en diversos lugares del mundo exceptuando aquéllos donde, por factores religiosos, el porcino no se incluye dentro de la dieta. Sus distribuciones se sobreponen y sus prevalencias son difíciles de establecer (2).

En las Américas sólo las islas del Caribe no han registrado cisticercosis porcina. En Brasil, que tiene más del $65 \%$ del total de porcinos de América Latina, se registró una tasa de infección por larva de $T$. solium del $0,83 \%$ en 12 millones de porcinos sacrificados en 10 estados durante el trienio de 1970-1972. Las tasas de infestación en países latinoamericanos varían del 1,35\% en Panamá, $2,57 \%$ en Honduras, $4.8 \%$ en Guatemala, $0,5 \%$ en Uruguay y $0,01 \%$ en Colombia y Chile (3).

En Colombia, se ha reportado el 2.,6 y el 7,5\% de prevalencia de cisticercosis porcina paralos mataderos de Cáqueza (4) y Cúcuta (Corredor A., comunicación personal) respectivamente. Las muestras de suero analizadas para el primero fueron de 228 en 1985 y de 265 para el segundo en 1990.

El diagnóstico clínico y parasitológico de cisticercosis porcina se realiza en pie mediante un examen semiológico, haciendo inspección y palpación digital de las conjuntivas oculares, base de la lengua y mucosa sublingual, zonas en las que son visibles o palpables los cisticercos (5) y también por el aumento del tamaño de los hombros (6).

El diagnóstico de cisticercosis porcina también se hace por la inspección de carnes en los mataderos (7). En éstos y plazas de ferias, se encuentran personas diestras que con alguna seguridad al examinar la lengua del porcino vivo, diagnostican la enfermedad.

La inspección sanitaria en el matadero se realiza después del sacrificio del animal, la cual se inicia en la parte externa de la canal y se finaliza en sus partes internas.

No se utilizan las técnicas inmunológicas como rutina para el diagnóstico de cisticercosis porcina premortem (8). Sin embargo, algunas técnicas inmunodiagnósticas para la detección de anticuerpos contra larva de $T$. solium han sido reportadas: hemaglutinación indirecta (HI) (4), inmunoelectroforesis (9) y $\operatorname{ELISA}(8,10)$.
La cisticercosis porcina provoca graves pérdidas económicas, ya que los animales contaminados con cisticercos son decomisados en el matadero, y puede propagarse rápidamente en explotaciones rústicas en malas condiciones sanitarias e higiénicas, debido al mal manejo de excretas humanas por la ausencia o mala utilización de letrinas, usándose aquellas como abonos para cultivos de hortalizas o al hacerse accesibles a los porcinos de hábitos coprófagos $y$, en general, por el bajo nivel socio-cultural, agravado lo anterior por la falta de inspección veterinaria pre y postmortem.

Debido a estas circunstancias y con base en el estudio epidemiológico realizado en el municipio de Coyaima por el Servicio Seccional de Salud del Tolima y el Instituto Nacional de Salud en el cual se encontró una seroprevalencia de $12,4 \%$ de la población humana de la región como positiva para anticuerpos de larva de $T$. solium, se fundamenta y explica la necesidad de realizar un estudio para verificar la prevalencia de cisticercosis porcina en las veredas: Totarco Dinde, Totarco Piedras, Doyare La Esmeralda y Lomas de Guaguarco, pertenecientes a este municipio, estandarizando y evaluando para ello el ELISA que es un método simple, económico y confiable que permite su empleo en serodiagnóstico y seroepidemiología de enfermedades parasitarias, como es el caso que nos ocupa.

\section{MATERIALES Y METODOS}

\section{Localización del área de estudio}

El área de estudio se encuentra ubicada en el departamento del Tolima, jurisdicción municipal de Coyaima a $392 \mathrm{msnm}$, con una temperatura promedio de $26^{\circ} \mathrm{C}$, a los $3^{\circ} 38^{\prime}$ de latitud norte y a $75^{\circ} 12^{\prime}$ de longitud oeste; cubre un área de 698 $\mathrm{km}^{2}$ conformada por 52 veredas cuatro de las cualesfueron muestreadas: Totarco Dinde, Totarco Piedras, Doyare La Esmeralda y Lomas de Guaguarco. El área en general presenta una topografía ligeramente ondulada o plana en la mayor parte de su extensión y se ubica en la formación de bosque seco tropical de Holdrige.

Coyaima limita geográficamente por el norte con los municipios de Ortega y Purificación, al sur con Natagaima, al oriente con Purificación y Prado y al occidente con Ataco, Chaparral y Ortega. 


\section{Tamaño de muestra}

El tamaño de la muestra se determinó utilizando un muestreo aleatorio simple para proporciones o muestra estrictamente aleatoria del número de porcinos presentes en la región sin distingo de raza, sexo o edad.

Para determinar el tamaño de la muestra se usó la siguiente fórmula:

$$
\begin{array}{ll}
n=\frac{Z \alpha^{2} \wedge p \cdot \wedge q}{d^{2}} & =0.5 \\
\wedge p=\text { exito } & =0.5
\end{array}
$$

$Z_{\alpha}=$ distribución normal =1,96 constante al $95 \%$

$$
\begin{aligned}
& \mathrm{d}=\text { error del muestreo }=9 \% \\
& \mathrm{n}=\frac{(1,96)^{2} \times 0.5 \times 0.5=118,56}{(0,09)^{2}}
\end{aligned}
$$

Según el ICA, regional número 6 , la población porcina existente en las cuatro veredas muestreadas (Totarco Dinde, Totarco Piedras, Doyare la Esmeralda, Lomas de Guaguarco) del municipio de Coyaima durante el año de 1986 fue de 1350 animales.

$$
\begin{aligned}
& n=\frac{n}{1+(n / N)} \\
& \mathrm{N}=\text { número de porcinos } \\
& \mathrm{n}=\text { número de porcinos a muestrear } \\
& \mathrm{n}=\frac{118.56}{1+118,5 / 1350}=109 \text { porcinos a muestrear }
\end{aligned}
$$

El total de porcinos escogidos, mediante muestreo aleatorio simple para proporciones, en su hábitat natural fueron 117. De los cuales 23 vivían en Totarco Dinde, 45 en Totarco Piedras, 16 en Doyare La Esmeralda y 33 en Lomas de Guaguarco.

Los porcinos no se encontraban bajo régimen sanitario alguno; su alimentación consistía de frutos propios de la región (guayaba, mango, plátano) y ocasionalmente, casos excretas humanas.

\section{Muestras}

Las muestras de sangre se obtuvieron de porcinos en pie, por venopunción con aguja número 20 de la vena marginal de la oreja: se centrifugaron y el suero se distribuyó en alícuotas de 500 ul y se almacenó a $-20^{\circ} \mathrm{C}$.

\section{ELISA}

\section{Estandarización}

Se determinó la concentración óptima de antígeno y las diluciones óptimas de suero y conjugado.

El inmunoensayo se realizó siguiendo la metodología descritapor Vollery colaboradores en 1976 (11) yutilizando placas de poliestireno Dynatech Immulon I. Los pozos de las placas fueron adsorbidos con 100 ul de antígeno en concentraciones seriadas de 1,2,5, $5,10 \mu \mathrm{g} / \mathrm{ml}$ y cada una adicionada por triplicado diluida en buffer carbonato $0,05 \mathrm{M}, \mathrm{pH}$ 9,6. Después de tres horas de incubación en cámara húmeda a temperatura ambiente, las microplacas fueron lavadas tres veces consecutivas con solución reguladora de buffer fosfato (PBS) $0,15 \mathrm{M}$, pH 7,4 más Tween 20 al $0,05 \%$ y cada vez durante 5 minutos.

Se agregaron por triplicado 100 ul tanto de suero positivo como de suero negativo en diluciones seriadas de $1: 200,1: 400,1: 800$ en la microplaca. La reacción antígeno-anticuerpo se llevó a cabo a temperatura ambiente $\left(20^{\circ} \mathrm{C}\right) \mathrm{du}$ rante dos horas, tiempo al cual se realizó un proceso de lavado con PBS-Tween como se mencionó anteriormente.

El conjugado (Sigma Chemical Co. A1186 anti-pig lgG unido a la enzima fosfatasa alcalina) en diluciones seriadas de 1:3.000,1:6.000,1:9.000 se adicionó por triplicado en volúmenes de 100 ul por pozo a la microplaca e incubó durante 18 horas a $4^{\circ} \mathrm{C}$. Un lavado final se llevó a cabo con PBS-Tween de la manera descrita previamente.

La reacción enzima-sustrato se desarrolló adicionando $100 \mu \mathrm{l}$ de $\mathrm{p}$-nitro-fenil-fosfato a una concentración de $1 \mathrm{mg} / \mathrm{ml}$ a cada pozo e incuban- 
do a temperatura ambiente durante 30 minutos. Esta se interrumpió agregando 25 ul de $\mathrm{NaOH} 3 \mathrm{~N}$ a cada pozo de la microplaca.

Finalmente, la reacción antígeno-anticuerpo fue determinada mediante la lectura de absorbancia a $405 \mathrm{~nm}$ en un colorímetro UNISKAN I.

\section{Determinación del punto de corte}

El valor de absorbancia para diferenciar las muestras positivas de las negativas (sueros de porcinos libres de cisticercosis) se estableció calculando el promedio de los valores de absorbancia de las muestras de sueros negativos más dos desviaciones estándar $(p<0,05)$.

\section{Evaluación}

La evaluación se realizó utilizando sueros de porcinos clínica y parasitológicamente comprobados con larva de $T$. solium (controles positivos), sueros de porcinos con Ascaris suum pero negativos a cisticercosis (para determinar reacción cruzada o especificidad de la prueba) y sueros de porcinos encontrados negativos para parásitos después de la inspección en el matadero Carlima de Ibagué.

La sensibilidad (S), especificidad (E), valor predictivo positivo (VPP) y valor predictivo negativo (VPN) del ELISA se determinaron utilizando una tabla de contingencia de dos por dos.

\section{Determinación de la prevalencia}

Los valores de absorbancia de las 117 muestras estudiadas que fueron mayores o iguales al punto de corte se consideraron positivas a anticuerpos contra larva de $T$. solium.

La prevalencia para proporciones se estableció utilizando los intervalos de confianza del 95 y $99 \%(12)$.

\section{RESULTADOS}

\section{Estandarización del ELISA}

La concentración óptima de antígeno de larva de $T$. solium fue de $5 \mathrm{ug} / \mathrm{ml}$ y las diluciones óptimas de suero y conjugado fueron de $1: 400 \mathrm{y}$ 1:3.000 respectivamente.

\section{Determinación del punto de corte}

El punto de corte para el ELISA del estudio fue de 0,48 . Las muestras se consideraron positivas si la absorbancia era igual o mayor a la media más dos desviaciones estándar de los valores obtenidos para los porcinos control.

\section{Validación del ELISA}

La sensibilidad del ELISA fue del 100\%, la Especificidad del $97,14 \%$, el VPP del $90 \%$ y el VPN del $100 \%$ para $(p<0,05)$ (Tabla 1$).$

\section{Prevalencia de cisticercosis porcina}

La prevalencia de cisticercosis porcina en las cuatro veredas estudiadas se observa en la tabla 2.

Los intervalos de confianza al 95\% para las veredas se aprecian en la tabla 3

TABLA 1. Cisticercosis porcina Validación del Elisa

\begin{tabular}{ll|l|l|l}
\hline A & \multicolumn{4}{|c}{ sueros porcinos } \\
B & \multicolumn{2}{c}{ comprobados por diagnóstico parasitológico } \\
S & & + & - & \\
O & & + & 1 & 10 \\
R & $>0,48+$ & 9 & 1 & \\
B & & & & \\
A & & & & 34 \\
N & $<0,47-$ & 0 & 34 & 34 \\
C & & & & \\
I & & & & \\
A & & 9 & 35 & 44 \\
$405 \mathrm{~nm}$ &
\end{tabular}

S: $100 \%$ E: $97,14 \%$ VPP: $90 \%$ VPN: $100 \%$

TABLA 2. Prevalencia (\%) de cisticercocis porcina en el municipio de Coyaima, departamento del Tolima

\begin{tabular}{|c|c|c|c|c|c|}
\hline & $\begin{array}{c}\text { Totarco } \\
\text { Dinde }\end{array}$ & $\begin{array}{l}\text { Tortaco } \\
\text { Piedras }\end{array}$ & $\begin{array}{c}\text { Doyare } \\
\text { La } \\
\text { Esmeralda }\end{array}$ & $\begin{array}{l}\text { Lomas de } \\
\text { Guaguarco } \\
\text { a }\end{array}$ & $\begin{array}{l}\text { TOTAL } \\
\text { veredas }\end{array}$ \\
\hline$a / n$ & $4 / 23$ & $15 / 45$ & $6 / 16$ & $5 / 32$ & $30 / 117$ \\
\hline$\%$ & 1,39 & 33,33 & 37,50 & 15,63 & 25,64 \\
\hline
\end{tabular}

$\mathrm{n}$ : Muestra total de sueros recolectados a: Muestras de suero positivas 
TABLA 3. Intervalos de confianza para la prevalencia de cisticercosis porcina por vereda y para el total, Coyaima (Tolima), 1993

\begin{tabular}{|c|c|c|c|c|}
\hline \multirow[b]{2}{*}{ VEREDA } & \multirow[b]{2}{*}{$\begin{array}{l}\text { TAMAÑO DE } \\
\text { MUESTRA }\end{array}$} & \multirow[b]{2}{*}{$\begin{array}{l}\text { PROPORCION DE } \\
\text { POSITIVOS (\%) }\end{array}$} & \multicolumn{2}{|c|}{$95 \%$ DE CONFIABILIDAD } \\
\hline & & & $\begin{array}{l}\text { LIMITE } \\
\text { INFERIOR }\end{array}$ & $\begin{array}{l}\text { LIMITE } \\
\text { SUPERIOR }\end{array}$ \\
\hline Tortaco Dinde & 23 & $4 / 23=17,39$ & 1,90 & 32,88 \\
\hline Tortaco Piedras & 45 & $15 / 45=33,33$ & 19,56 & 47,10 \\
\hline Doyarela Esmeralda & 16 & $6 / 16=37,50$ & 13,78 & 61,22 \\
\hline Lomas de Guaguarco & 32 & $5 / 32=15,63$ & 3,05 & 28,21 \\
\hline \multirow[t]{2}{*}{ TOTAL } & 117 & $30 / 117=25,64$ & 17,73 & 33,55 \\
\hline & & & \multicolumn{2}{|c|}{$99 \%$ DE CONFIABILIDAD } \\
\hline VEREDA & $\begin{array}{l}\text { TAMAÑO DE } \\
\text { MUESTRA }\end{array}$ & $\begin{array}{l}\text { PROPORCION DE } \\
\text { POSITIVOS }\end{array}$ & $\begin{array}{l}\text { LIMITE } \\
\text { NFERIOR }\end{array}$ & $\begin{array}{l}\text { LIMITE } \\
\text { SUPERIOR }\end{array}$ \\
\hline Tortaco Dinde & 23 & $4 / 23=17,39$ & 0,00 & 37,78 \\
\hline Tortaco Piedras & 45 & $15 / 45=33,33$ & 15,20 & 51,46 \\
\hline Doyarela Esmeralda & 16 & $6 / 16=37,50$ & 6,27 & 68,73 \\
\hline Lomas de Guaguarco & 32 & $5 / 32=15,63$ & 0,00 & 32,19 \\
\hline TOTAL & 117 & $30 / 117=25,64$ & 15,23 & 36,05 \\
\hline
\end{tabular}

\section{DISCUSION}

La prevalencia del $25,64 \%$ de cisticercosis porcina en las veredas estudiadas del municipio de Coyaima es superior al $12,4 \%$ de prevalencia de cisticercosis humana encontrada por el Instituto Nacional de Salud y el Servicio Seccional de Salud del Tolima en 1990 para esta área. Esto permite observar que la cisticercosis humana y porcina evidentemente se sobreponen en esta región.

La prevalencia encontrada en este estudio está más aproximada a la realidad que la encontrada en mataderos ya que las muestras fueron tomadas en porcinos en pie, en su hábitat natural $y$ en condiciones de manejo propias de sus dueños. Muchos de los animales no llegan a los mataderos, pues, existen comerciantes diestros en detectar la enfermedad y, por lo tanto, no adquieren los animales enfermos, los cuales son sacrificados y consumidos en las veredas.

Lo anterior puede ser una posible explicación a los valores inferiores de prevalencias reportadas en los estudios realizados en mataderos de Cáqueza, Cundinamarca, y Cúcuta, Norte de Santander.

La prevalencia de cisticercosis porcina varía entre los países latinoamericanos desde el $0.01 \%$ al $4.8 \%$ (4). Este fenómeno no sólo se observa entre países sino también entre regiones de un mismo país como es el caso de Colombia donde Cáqueza, Cúcuta y Coyaima presentaron prevalencias del $2,6 \%, 7,5 \%$ y $25,64 \%$ respectivamente; este suceso es repetitivo entre áreas 
de una misma región tal como fue observado en las cuatro veredas estudiadas, Totarco Dinde $(17,3 \%)$, Totarco Piedras $(33,33 \%)$, Doyare La Esmeralda $(37,5 \%)$ y Lomas de Guaguarco $(15,63 \%)$.

Las diferencias encontradas entre los paises podrían explicarse por las condiciones existentes tanto en el acceso a servicios públicos y sanitarios como por el grado de tecnificación en las explotaciones porcinas y las diferencias entre las veredas podrían deberse no sólo a la idiosincrasia de los habitantes sino también al grado de desarrollo socioeconómico y cultural presentes en cada una de ellas.

Es de primordial importancia para médicos y médicos veterinarios el mantenimiento del buen estado de salud humana y porcina respectivamente. Si este personal no es conciente de los problemas que causa la cisticercosis, las consecuencias proseguirán siendo funestas ya que las personas pueden seguir manteniendo el ciclo de vida del parásito en su sistema digestivo (teniasis) y mediante la eliminación de los huevos al medio ambiente continuar así no sólo con la cisticercosis sino también con la neurocisticercosis, que acarrean la incapacidad humana a nivel físico y laboral así como el decomiso de los ejemplares en los mataderos, afectando el poder adquisitivo del campesino y produciendo pérdidas económicas en la región.

Esta investigación puede ser el punto de referencia para establecer un estudio seroepidemiológico de anticuerpos contra larva de $T$. solium tanto en porcinos como en humanos a nivel departamental y nacional para instituir las medidas de control necesarias en forma inmediata en la región ya estudiada y posteriormente extrapolarlas a otras áreas del país donde la cisticercosis sea endémica.

El control de la cisticercosis debe encaminarse a la educación de la comunidad, por intermedio de los líderes comunales que pueden llegar en forma directa y efectiva a su gente. El adiestramiento de éstos deberá estar orientado a:

- Un adecuado manejo de excretas mediante la construcción de letrinas que evitan la contaminación de las aguas de riego de los cultivos con las excretas humanas y disminuyen el riesgo de infestación del porcino, dados sus hábitos coprófagos.

- Evitar que las materias fecales sean utilizadas como abonos en los cultivos.

- Impulsar la construcción de porquerizas y corrales para evitar el contacto de los porcinos con excretas humanas.

- Promover entre la población los buenos hábitos higiénicos.

Fomentar la correcta cocción de los alimentos y el perfecto asado o fritura de las carnes.

- Establecer por medio de las entidades gubernamentales la construcción de mataderos municipales que permitan el sacrificio de los porcinos de la región bajo la adecuada inspección veterinaria.

\section{SUMMARY}

One hundred and seventeen sera of alive pigs in rural areas from the counties of Totarco Dinde, Totarco Piedras, Doyare La Esmeralda and Lomas de Guaguarco, belonging to the municipality of Coyaima in the department of Tolima, were obtained during the period from June to December in 1992 and analyzed for antibodies against larvae of Taenia solium by enzyme linked immunosorbent assay (ELISA) in order to establish the prevalence of cysticercosis in pigs.

The ELISA test for immunodiagnosis of cysticercosis in pigs was standardized. The optimum concentration of total delipidized antigen of $T$. solium larvae was $5 \mu \mathrm{gr} / \mathrm{ml}$ and the optimum dilutions of serum and conjugate (anti-pig IgG linked to alkaline phosphatase) were 1:400 and $1: 3000$ respectively.

The parameters of the test were: sensitivity: $100 \%$, specificity: $97.14 \%$, positive predictive value: $90 \%$ and negative predictive value: $100 \%$, using serum samples from healthy pigs and swines having a parasitologically confirmed diagnosis of cysticercosis. 
Prevalence of cysticercosis in pigs from the municipality of Coyaima was $25,64 \%$, being $37,5 \%$, $33,33 \%, 17,39 \%$ and $15,63 \%$ for the counties of Doyare La Esmeralda, Totarco Piedras, Totarco Dinde and Lomas de Guaguarco, respectively.

The ELISA test carried out in this survey might be applied for both immunodiagnosis of cysticercocis in pigs and seroepidemiological surveys of prevalence of the disease in swines in order to set up control measures against this parasite.

\section{AGRADECIMIENTOS}

El presente estudio fue auspiciado por el Instituto Nacional de Salud (INS) y el Servicio Seccional de Salud del Tolima (SSST).

A los mataderos Distrital Central de Santafé de Bogotá y Carlima de Ibagué.

A los doctores Ramiro Rodríguez, bacteriólogo, Jefe, Red Seccional de Laboratorios del SSST y Edgar Alfredo Saavedra Campos M.V, M.Sc. economía; a los señores Alvaro Galindo y Miguel Rodríguez por su desinteresada colaboración.

A la comunidad del área estudiada y al personal del Hospital San Roque de Coyaima.

\section{REFERENCIAS}

1. Malagón F. Elementos del binomio teniasis/cisticercosis: una sintesis. En: Flisser A, Malagón $F$, eds. Cisticercosis humana y porcina. México: Limusa, 1989: 3-6.

2. Abdussalam M, Gemmel MA, Griffiths RB, Grissklaus $D$, Kagan IG. Recherches necessaires en matiere de Taeniase cysticercose. Bull WHO 1979; 53: 371-8.
3. Acha P. Zoonosis y enfermedades transmisibles al hombre y a los animales. Washington: OMS, 1977 : $501-3$

4. Molano P. Estudio de prevalencia de cisticercosis porcina en el municipio de Cáqueza. Tesis de grado. Villavicencio, Meta: Universidad Tecnológica de Los LLanos Orientales, 1985; $73 \mathrm{p}$.

5. Contreras L. Aspectos más sobresalientes de la inspección sanitaria de los suinos en los rastros del D.F. y en otros del pais en busca del cisticerco de Taenia solium. En: Flisser A, Malagón F, eds. Cisticercosis humana y porcina. México: Limusa, 1989; $257-9$

6. Rodríguez J, Meneses M. Ensayo farmacológico del praziquantel (droncit) sobre Cysticercus cellulosae en porcinos. Ibagué: Universidad del Tolima, 1985.

7. Aluja AS. Frequency of porcine cysticercosis in Mexico. En: Flisser A, Willms K, Laclette JP, Larralde C, eds. Cysticercosis: Present state of knowledge and perspectives. New York: Academic Press 1982; 53-62.

8. Torres LA. Evaluación del efecto de diferentes dosis de praziquantel en cerdos parasitados naturalmente con el metacestodo de la Taenia solium. Master in Veterinary Science, Thesis. Mexico, DF. Faculty of Veterinary Medicine, National University of Mexico, 1990; 106.p.

9. Pathak KM, Gaur SN, Garg SK. Counter-current immunoelectrophoresis, a new technique for the rapid serodiagnosis of porcine cysticercosis. 1 Helminthol 1984; 58: 321-4.

10. Gómez LF, Mora E. Estandarización y evaluación de la técnica de ELISA para el diagnóstico de la cisticercosis porcina. Tesis de grado. Bogotá, Cundinamarca: Universidad Nacional de Colombia, 1988; 91.p.

11. Voller A. Enzyme immunoassays for parasitic diseases. Trans R Soc Trop Med Hyg 1976; 70:98

12. Wayne D. Bioestadística. México: Limusa 1980: 128130 